\title{
The "enhancing tertiary tutor's cultural safety" study: Aboriginal and Torres Strait Islander cultural training for tutors of medical students
}

\author{
F. Merritt ${ }^{1}$, J. Savard ${ }^{2}$, P. Craig \& A. Smith $^{1}$
}

\begin{abstract}
Introduction: The inclusion of an Aboriginal and Torres Strait Islander health curriculum is an accreditation requirement of medical training. Healthcare provisions that are culturally safe may lead to better health outcomes for Aboriginal and Torres Strait Islander peoples. To achieve this, graduating doctors need to practise in a culturally-safe manner when dealing with Aboriginal and Torres Strait Islander Australians. This study considered the impact of a cultural diversity/cultural-safety training program for problem-based learning (PBL) tutors of medical students.
\end{abstract}

Methods: This qualitative study looked at the impact of a cultural-safety training workshop that was delivered to current PBL tutors of medical students. The tutors were invited to participate in a focus group 6 months post workshop and asked to reflect on the content and structure of the workshop, their role as a tutor and whether their workshop experience translated to their tutorial work.

Results: The three key thematic findings from the focus group were around reflections on the tutor's own cultural competence, the perceived role of the tutor in the PBL environment and the interaction between the tutor and the student in their tutorial groups. Interestingly, across these findings, an "us versus them" construct was noted.

\footnotetext{
1 School of Medicine, Sydney, The University of Notre Dame, Australia

2 School of Medicine, Faculty of Health, Deakin University, Australia

\section{Correspondence}

Associate Professor Frankie Merritt, PhD

Head of Aboriginal and Torres Strait Islander Health

The School of Medicine, Sydney

The University of Notre Dame Australia

160 Oxford Street, Darlinghurst

Sydney

Australia

Tel: +61282044456

Email: frankie.merritt@nd.edu.au
} 
Conclusion: PBL tutors can be agents of change; they are uniquely positioned to enable future doctors to have a pivotal role in better health outcomes for Aboriginal and Torres Strait Islander people. We found a single cultural-safety training event to be helpful but insufficient. Addressing systemic barriers towards institutional change across health professional education is required.

Keywords: Indigenous; Aboriginal; higher education, students; awareness; cultural safety; racism; culture; problem-based learning; medical curricula.

\section{Introduction}

Australia is a complex and culturally-diverse nation. As such, health professionals must be competent practitioners who are aware of cultural diversity, psychological factors and social context, all of which impact upon health. An awareness of these perspectives encourages a broader view of health, one that acknowledges the sociocultural determinants of health alongside the biomedical view. However, barriers to effectively addressing and teaching these factors within medical curricula still remain.

The Australian Medical Council (AMC) is empowered to accredit medical schools via its accreditation processes (Australian Medical Council, 2017). To be accredited, Australasian medical schools are required to be compliant with standards around their curriculum coverage of Indigenous health and are required to address cultural awareness and diversity (Australian Medical Council, 2017). In this endeavour, they are guided by key elements of the Indigenous Health Curriculum Framework, developed by the Committee of Deans of Australian Medical Schools (now Medical Deans), which includes learning outcomes related to Aboriginal and Torres Strait Islander health and to the monitoring, evaluation and delivery of the curriculum (Phillips, 2004). Despite these mandates, a gap remains between the health and life expectancy of Aboriginal and Torres Strait Islander Australians and non-Indigenous Australians (Gladman, Ryder, \& Walters, 2015). Culture is a notoriously difficult term to define but most commonly is epitomised by terms such as beliefs, values, habits and rituals (Culture, 2018). Aboriginal and Torres Strait Islander patients are more likely to access health services where practitioners communicate respectfully, have an understanding of culture, can build relationships with Aboriginal and Torres Strait Islander patients and where Aboriginal or Torres Strait Islander health workers are included in the treating team (Commonwealth of Australia Department of Health, 2014). As a result, care that is culturally safe is more likely to increase better health outcomes for Aboriginal and Torres Strait Islander peoples (Commonwealth of Australia Department of Health, 2014). To achieve this outcome, graduating doctors need to be culturally aware and operate in a culturally-safe manner when dealing with Aboriginal and Torres Strait Islander Australians. However, it is important to acknowledge that any health professional is only a part of a system. The evolution of any changes, from research findings, to policy change and to subsequent curriculum change, involves a significant time frame.

Although medical schools are guided by the accreditation process and the various frameworks to assess how cultural content is enacted in the curriculum, delivery of Aboriginal and Torres Strait Islander content in the curriculum is not easy. Current 
research on different approaches reports how challenging it is to sculpt curricula in a way that can translate into future better health outcomes for diverse cohorts (Durey, 2010). Challenges include learner resistance, the perpetuation of stereotypes and "othering" and the lack of focus on the learner (Boutin-Foster, Foster, \& Konopasek, 2008). Research reporting on student understanding of their tutor's perspectives of curricula that focus on race, ethnicity and culture found that despite students rating diversity as important, they did not consider the subject matter to be relevant to biomedicine (Roberts, Sanders, Mann, \& Wass, 2010). Students adopting their tutors' perspectives on diversity, race and ethnicity may demonstrate the degree to which students "conform to the institution's dominant values in order to succeed" (Roberts et al., 2010, p. 559). This is reflective of a professionalised socialisation process-where despite increasing diversity in medical school cohorts, a uniformity of values, attitudes and practice is still developed within medical graduates (Beagan, 2000).

Approaches to diversity, cultural-safety and cultural-awareness training tend to target large groups of people, rather than taking a more individualised approach. Cultural education has a role to play in addressing inequities in Aboriginal health, but cultural awareness itself is not enough. Generally, cultural awareness can be defined as attempts to increase peoples' awareness of factors such as cultural, societal and historical factors around those who are not of the dominant culture. Part of this awareness includes encouraging participants to challenge stereotypes and focus on self-reflection on the participant's own culture (Downing, Kowal, \& Paradies, 2011). Downing et al. (2011) highlighted a theoretical view that cultural-awareness models may not lead to lasting change but may, instead, encourage "othering" (Downing et al., 2011). This is in part due to a lack of focus on the diversity within Indigenous Australia, a focus on "indigenous culture" (in a way that is limited in its concepts of identity and culture) and a lack of a focus on broader health services and systems. Cultural safety, in contrast, explicitly deals with power, systems and inequity and, therefore, has better potential to create culturally-safe health systems (Downing et al., 2011).

For this study, we were specific in our approach, targeting the tutors of problem-based learning (PBL) tutorials with a cultural diversity training program, for one medical school. PBL tutors, who are also clinicians, have the most access to medical students in their early training years. They see the students the most in face-to-face hours in small group settings. This approach also introduces cultural safety and training in a way that engages the key people who fulfil a role in the day-to-day implementation of the specified medical curriculum.

During the first 2 years of a 4-year medical degree at the School of Medicine, Sydney Campus, the University of Notre Dame Australia, students are taught in small groups using a PBL approach. PBL tutorials are structured around a case-based scenario with student-led learning, supported with facilitation by tutors (Azer, 2007). The School of Medicine, Sydney Campus, is committed to a focus on Aboriginal and Torres Strait Islander health and meets requirements through several means_-one of which includes PBL scenarios with an Aboriginal and Torres Strait Islander focus. Therefore, an 
essential skill for PBL tutors is the ability to engage, facilitate and respectfully explore culturally-diverse scenarios.

PBL tutors need to be culturally aware to adequately explore and successfully undertake this type of debate and learning around diverse populations. To increase the competence of the targeted tutor group, an organisation was identified to deliver a workshop on cultural safety. This organisation was chosen as it promotes the requirements for intercultural competence to be balanced across four vital components: knowledge (about other cultures and other people's behaviours), empathy (understanding the feelings and needs of other people), self-awareness (knowledge of one's desires, strengths, weaknesses and emotional stability) and cultural identity (knowledge of one's culture). The cultural training workshop delivered is accredited by the Royal Australian College of General Practitioners (RACGP) and was specifically targeted at PBL tutor groups.

This study sought to investigate the impact of a cultural diversity training program when delivered to PBL tutors of medical students. In this paper, we report the impact a cultural-safety training program had on PBL tutors in an Australian medical school.

\section{Methods}

The methodology was qualitative, incorporating thematic analysis. It was evaluative in nature, and it incorporated immediate and longer-term evaluation. Bias was mitigated by team meetings to monitor coding, review coding and thematic findings and discuss possible findings. The study explored the impact of a cultural-safety training workshop delivered to current PBL tutors of medical students. The program lasted for half a day and covered a broad range of issues-starting with explanations of Aboriginal and Torres Strait Islander culture and an abbreviated history of Aboriginal and Torres Strait Islander peoples, followed by activities highlighting the role and meaning of culture and concluding with a question and answer period. All 18 tutors for the 2015 academic year attended the workshop.

The workshop was delivered prior to the start of the 2015 academic year, and 6 months after the workshop, the tutors who attended were invited to participate in a focus group, where they were asked to reflect on the content and structure of the workshop, their current role as a tutor and whether their workshop experience translated to their work during the first semester of teaching. Ethics approval was granted by the University of Notre Dame Australia's Human Research Ethics Committee (Ethics Approval Number 015010S) prior to the research commencing.

Two dates during the mid-semester break were offered for focus groups to accommodate the tutors' schedules. Due to a misunderstanding, no tutors attended on the first date, but eight participated in a focus group facilitated by the research assistant at the subsequent date; a separate telephone interview was conducted with an additional tutor. The same research assistant (RA) moderated the focus group, conducted the interview and was also present at the workshop. Thus, when issues were raised by participants about the workshop, the RA was able to prompt and ask participants to expand on certain points of reference. 
While interviews and focus groups involve different dynamics between the researcher and the participants, for this research, the two methods were complimentary, as the RA worked from an identical focus group/interview schedule. Thus, the focus group data informed questions the RA asked in the interview, and the RA was able to raise issues and questions presented in the focus group. This overall approach enabled the research team to explore issues further and to collect rich data. This approach was also useful pragmatically, as it allowed the research team to include participants who were unable to attend the focus group, thereby expanding the attendance and participation in the research itself.

The focus group and telephone interview were recorded and transcribed verbatim. Transcripts were validated by the RA; identifying information was removed; and the transcripts were analysed using a thematic analysis approach, as outlined by Braun and Clarke (2013). In this case, the transcripts (from the focus group and the interview) were initially reviewed by the RA for content, followed by a detailed line-by-line coding. From this, a summary table was constructed, with possible themes identified. A meeting was then held with the research team, where themes were refined and ideas and concepts to be explored further identified. A second coding was conducted by the RA to produce a summary of the findings from the focus group.

\section{Results}

The focus group and interview highlighted the roles assumed by tutors, the roles students adopted and how tutors and students interact when faced with tension around cultural issues. Three key thematic findings from the focus group/interview are noteworthy: first, the ways in which tutors could describe their own cultural competency as they reflected on their experience at the workshop earlier in the year; second, how the tutors perceived their relationship with their students functions and their role in the PBL tutorial; finally, how the interaction between the students and the tutor reinforces their assumed roles and together gave rise to adaptive strategies used by both tutors and students when dealing with culturally-sensitive issues or situations. Across these findings, a construct of "us versus them" was found to be present. This construct is important because it demonstrates the ways in which both tutors and students orient themselves to identify and, in some cases, avoid culturally-complex or challenging issues.

\section{Self-reflection by the tutors on their own cultural competence}

When tutors talked about their experience of the workshop 6-months prior and what they remembered, there was a strong focus on the ways in which the facilitator brought attention to the importance of culture. This was demonstrated through tutors' reflections on the workshop introduction session, where both the main facilitator and the support facilitator talked about their own Aboriginal culture and identity and the importance of these to them. The tutors were then invited to participate in a round-table introduction of their own cultural backgrounds to the group. On reflection, some tutors voiced their appreciation of this exercise, as it enabled them to reflect on what their own culture meant and how it impacted upon how they positioned themselves in the world. As one tutor reflected: 
Talking about our own culture, I thought, was a really useful exercise, because you suddenly realise ... if you don't reflect on it yourself, it's hard to work out what it means for somebody else to identify with culture. (Participant 9)

Later, the same tutor continued:

I think just having ... the opportunity for people to ... talk and air their opinions made me think a bit harder about my own opinions and prejudices and ... how I felt about ... what other people said and their comments was helpful for me to ... reflect on, and I suppose that just made me a little bit clearer about what I think about the issues and so that, then, I suppose helps you to draw those ideas out from the students as well. (Participant 9)

When the tutors were asked to specifically reflect upon this exercise in the focus group, many identified that they felt the exercise (reflecting on their own culture) was beneficial. However, when reflecting on their experience of the workshop overall, they did not identify the connection between how they identify with their own culture and how that applies to their role as a tutor and clinician. In other words, they perceived culture to be about (and important to) others but not themselves. This was particularly salient when tutors reflected on the question and answer period of the workshop. For example, one tutor felt they did not get the answer they were expecting or wanted from the facilitator.

Can I play devils' advocate and just say that from the cultural awareness [workshop], I didn't get a lot of that, out of that cultural awareness.... I would have liked to have got a lot more about ... how Aboriginal health is affected or dealt with and how we should approach it, because of their culture. And I don't feel that I got that out of it. (Participant 4)

Therefore, it was clear that at least some tutors wanted specific answers and specific strategies to take into the classroom, seeing self-reflection as either superfluous or not "core business". This was not a view held by all tutors, as some participants felt the question and answer session at the end was the most useful part of the workshop.

I think the best part of that workshop was almost the bit at the very end, when we were all sitting in the circle and we were all able to ask questions [of both facilitators], and [they were] very open and honest [in responding] to those questions. (Participant 3)

The differing responses to a shared activity highlight the nuanced and complex ways culture can be interpreted, experienced and understood. Importantly, in the focus group, culture was not explicitly mentioned by the participants. When the workshop facilitator talked about culture, he talked about Aboriginal and Torres Strait Islander culture, but the tutors were resistant to and did not name their own culture as a situated perspective from which they viewed and interpreted the world. Noticeably, when tutors talked about how they identified, explored and confronted culturally-sensitive issues in the tutorials, they did not acknowledge their own culture and how it might shape their own perspective as a tutor or clinician. 


\section{The perceived role of the tutor in PBL tutorials}

When engaging with students, tutors talked about two different ways in which they would engage with their students about culturally-sensitive issues. First, they would adopt a passive and incidental approach to learning opportunities. With passive and incidental learning, the tutors would allow their students to "fall over", that is, to let their students adopt discriminatory language and use the opportunity to engage the students.

I think they get a lot out of those-just those really brief moments, when people are unintentionally ... discriminatory, where you can just bring a little bit of something in there in that moment and move on. (Participant 7)

Tutors were adept at using these instances of discomfort as learning opportunities for the students. Missing from these moments is the opportunity for the tutors (who are also clinicians) to remind their students that as a clinician, their own culture will assume a role in their interactions with patients.

The second way tutors would respond to students is by actively challenging and confronting them. In this instance, the tutor repeated the introductory exercise, from the workshop, with their students, such that they opened their tutorial by having their students reflect on their own cultures. Using this exercise, the tutor was able to frame the ways in which they wanted their students to approach culturally-sensitive issues from the beginning.

In the introductory PBL [tutorial], I asked [the students] questions about ... where they come from and their families and that kind of stuff, just to find out a bit more about them,... which they found really helpful, and I found really helpful as well. ... I think I probably do push a little bit harder, in terms of getting them to articulate some of their underlying beliefs about health and probably about culture and stuff as well. (Participant 9)

Tutors also viewed their role within the tutorial as being responsible for directing active learning. To do this, tutors would identify and label culturally-sensitive situations and instances, thereby opening them up for discussion.

It seems the responsibility of the tutor to, in general, to ... comment about ... inappropriate comments. I think it's, from my point of view, for my students ... in my group, definitely, if someone says something that is ... discriminatory towards Aboriginal health, then it's very rare that one of the other students says anything. (Participant 5)

While it is positive to observe that this tutor put what they learned from the workshop into practice in their PBL group, only one tutor acknowledged using this practice within their tutorials. This suggests tutors still have barriers to engaging and encouraging students to address and unpack culturally-sensitive issues.

\section{The interaction between the tutor and the student}

While an inherent part of the relationship between tutors and students frames the tutor as a facilitator, there is ample opportunity for tutors to influence and gently direct the learning of students. For example, when talking about students in the focus group, the 
tutors focused on how students' cultural biases affect how they understand and deal with culturally-sensitive issues.

My group has a little bit more variety in the background of the students. ... Although they are ... all Australian ... and from a mix of both rural and urban backgrounds,... there's a little bit of a spread of age and ethnic backgrounds of parents. And, I think that a lot of my students have had some time to reflect on their own cultural heritage. And so, they, sort of, bring to that a little bit more of what it's like to be the other. (Participant 2)

Unfortunately, one consequence of this structure is that tutors could perceive their students as "other". In this construct, tutors can identify the cultural bias the students demonstrate and address the behaviours in the student. However, tutors may do this while neglecting to acknowledge and claim their own biases in their interactions with the students (and as clinicians). This was reflected in how culture was talked about; it was understood to be an external other, and in some circumstances, tutors did not seem to apply culture to the themselves. This led to a missed learning opportunity for both the tutors and the students. Specifically, students may miss out on critical guidance and leadership from tutors and the opportunity to learn from the tutors' experience as a practising clinician. This, in turn, has implications for how students learn to deal with diversity and culturally-challenging issues in PBL tutorials (and potentially during GP placements) and how they are likely to respond when they themselves become clinicians.

\section{Discussion}

Our research focuses on medical PBL tutors and their role in translating culturally-safe knowledge to their students. This study aimed to explore the impact a cultural-safety training program had on PBL tutors in an Australian medical school. Our findings suggest that while the tutors in this study are aware of their own cultural identity, they may be resistant to acknowledging it when dealing with their students. However, a key perspective missing from the tutor group as a whole, and from their engagement with the students, was their own stance-their own cultural position and identity and how it might impact upon their position and view as a tutor and as a clinician. This resistance reflects their comfort (or discomfort) in dealing with culturally-sensitive issues and, consequently, impacts upon the role and approach they assume in PBL tutorials when such issues arise. Alternatively, individuals may not acknowledge their cultural perspective because it is taken for granted.

The PBL tutor assumes a central role in tutorials. They facilitate discussion and guide and encourage students to critically reflect upon their values and roles and the knowledge that shapes them as potential clinicians. This role is especially important when identifying, defining and dealing with culturally-sensitive issues. During the 6 months post workshop and as they reflected on their role as tutors, most participants in this study did not appear to address how their own cultural context and perspective might assume a role in the facilitation of their tutorials. It is important to acknowledge Participant 9, who was quoted in the results. To paraphrase this participant, if you don't reflect on your own culture and identity then how do you work out what culture and identity means 
for someone else? Self-reflexivity has been identified as a key component of learning and developing competency when working cross-culturally (Bringle, Hatcher, \& Jones, 2011). While the tutors in this study may practise self-refection outside of this study, their limited engagement in it within the context of cultural diversity and the training they are expected to impart to their students is cause for concern.

Participants in this study perceive their role in PBL as facilitation and would be guided by their reading of the group and each individual participant when deciding whether to allow issues to arise or how to facilitate if students experienced discomfort. The two approaches tutors talked about using, either an active or incidental role, are useful teaching tools that enable the tutor to guide students through their assessment and unpacking of the issues. However, missing from this engagement is the experience of the tutor and how they would deal with such issues as a clinician. By leaving themselves out of the discussion, the tutors are situating the issue as one belonging to the students—an "other" — which is how culture was talked about by both students and tutors. This is characteristic of the process of "othering", which is reported in the literature and has been identified as a barrier to successful transmission of knowledge and a barrier to instilling change in systems (Durey, 2010; Boutin-Foster et al., 2008). Unfortunately, in tutorials, this framing also arose in regards to how culturally-sensitive issues were identified by students and was reflected in the relationship between the tutor and the student.

While the relationship between tutors and students is organised in a particular way, this can often be a barrier in and of itself to translating knowledge. When it came to issues of cultural sensitivity, there seemed to be a particular distancing between the tutor and the student. As a result, the opportunity for students to learn from the tutor is greatly diminished or absent. It is important to note that the role of the PBL tutor is different from a traditional teacher/student dyad. The PBL tutor acts more as a facilitator of the learning process. Together with the high level of contact between student and tutor, the facilitator role offers a good model for encouraging empowerment and reflection on such complex topics as developing cultural sensitivity. The PBL tutor role is complex and nuanced. Tutors seek to promote clinical reasoning, information-seeking skills and interpersonal competencies such as communication. For example, when talking in the focus group, the tutors would focus on the students and how their cultural biases have a role to play in understanding and dealing with culturally-sensitive issues. As a consequence, students may learn how to navigate cultural difference based on how tutors approach difference. From the focus group, while tutors described approaching culturally-sensitive issues in a respectful manner, they did so by excluding themselves from the dialogue. Because of this, the students may learn to treat cultural issues as something belonging to their future patients, with their own culture absent from their view of their role as a clinician. This is problematic, because it reflects another barrier, a limited or absent acknowledgement of the effect of students' own culture on their education, which has the potential to translate into similar practices when students become practising clinicians.

While this type of separation and role delineation may serve as a form of adaptive strategy, acting as a buffer used by both tutors and students when dealing with culturally-sensitive issues or situations, it becomes disruptive and problematic and reflects a systemic and 
institutional issue. "Othering" is a useful concept to apply to this situation because it provides a way to conceptualise interpersonal and institutional structures that exist as hurdles to introducing cultural awareness. For teachings around diversity, cultural awareness and cultural safety, "it is essential that students see what they are being taught actually being practised by their clinical teachers" (Beagan, 2003, p. 614).

When students do not see clinicians modelling practices that attend to the cultural and social diversity of patients and practitioners, they begin to see socially-responsive medicine as, "all very nice to talk about in theory, but ultimately it makes no difference" (Beagan, 2003, p. 614). For example, the distance tutors create between their own cultural experiences and (perhaps) their patients' culture and diversity or their students' culture or diversity exhibits to students ways in which they might be expected to act when they become clinicians. This practice may become a barrier, preventing (and potentially halting) any opportunity for the tutor to demonstrate (and therefore model) why it is important to be critically aware of and reflect upon one's own culture before they are able to adequately acknowledge and address another's culture, which is the core of a culturallysafe approach.

Cultural diversity training needs to exist as an ongoing learning exercise for health professionals, requiring meaningful engagement from not only those facilitating it but also those learning. Critical reflection of self appeared to be a limited but fundamental practice needed by the tutors. Critical reflection is a vital tool to promote learning that is transformative, leading to a "socially informed, critically reflective practitioner" (Bennett, Power, Thomson, Mason, \& Bartleet, 2016, p. 3). Therefore, practitioners need to be reflexive and prioritise an honest and deep understanding of what they bring to the consult room (or indeed the classroom). Until doctors see their own biases, how can they see how they may impact on the consult room dyad? As Beagan (2000) said, "Until physicians learn to see their own biases, they will not be able to recognize their impact in encounters with patients; such recognition is an essential step toward substantive equality in health care" (pp. 1263-1264). Overcoming barriers and challenges to cultural awareness starts with enabling competency in the facilitators/educators in a medical school. Tutors were selected as a target cohort for cultural-safety training, and given the findings from this study, they were an appropriate target. PBL tutors have the most interaction and engagement with students early in the program and, thus, the most opportunities to teach and demonstrate to students ways they could approach the subject of cultural safety and culturally-competent practice. In this capacity, PBL tutors can become agents for change; their role is uniquely positioned to help future doctors enable better health outcomes for Aboriginal and Torres Strait Islander people. If they are teaching, discussing and modelling culturally-safe practices, then the goals of better health outcomes for Aboriginal and Torres Strait Islander peoples are achievable. 


\section{Limitations of this study and future research}

This study was qualitative in nature and, therefore, did not set out to be representative. Therefore, the findings may not be generalisable across other professions or from outside of the Australian context. The cohort was homogeneous, and the tutors were recruited from one campus of one Australian university. The response rate could be enhanced in future research, and this study could be used as a base for either a quantitative or mixedmethod study that utilises a larger and, therefore, more diverse sample of PBL tutors.

\section{Conclusion}

This study focused on tutors, but a key facet and focus of cultural safety is systems and power differentials. It cannot be left up to one part of a system to solve broader issues. It is important to state that while our study highlighted a systemic issue within one medical school, this is in no way an indictment of our tutors or of PBL tutors in general; rather, it suggests that the issue is likely to be found elsewhere in health professional education. Of concern is the potential lost opportunity of contributing towards culturally-safe health systems (Downing et al., 2011) and, thus, improved health outcomes for Aboriginal and Torres Strait Islander peoples (indeed, improved health outcomes for any diverse cohort). We found a single cultural-diversity training event to be helpful but insufficient. What is required is a strategy that works on systemic barriers towards institutional change across health professional education (Boutin-Foster et al., 2008; Durey, 2010; Laverty, McDermott, \& Calma, 2017).

Cultural diversity training has to be introduced in a way that works with systemic structures but also enables knowledge to be transferred, enriched and built within current structures. In general, tutors need to be on their own journey of cultural-diversity awareness and respect, as well as modelling such behaviour for their students. This modelling of reflexivity could encourage critical reflection in students. As a key stakeholder, tutors are central to training future clinicians, and they remain the best target for culture awareness and training, especially when their experience and knowledge as a clinician is combined with critical reflection skills they can share and demonstrate to students, thereby enabling the next generation of clinicians to be culturally competent and aware.

\section{References}

Australian Medical Council. (2017). Accreditation and recognition. Retrieved from https://www.amc.org.au/accreditation

Azer, S. A. (2007). Navigating problem-based learning. Marrickville, NSW, Australia: Elsevier.

Beagan, B. L. (2000). Neutralizing differences: Producing neutral doctors for (almost) neutral patients. Social Science \& Medicine, 51, 1253-1265.

Beagan, B. L. (2003). Teaching social and cultural awareness to medical students: "It's all very nice to talk about it in theory, but ultimately it makes no difference". Academic Medicine, 78(6), 606-614. 
Bennett, D., Power, A., Thomson, C., Mason, B., \& Bartleet, B.-L. (2016). Reflection for learning, learning for reflection: Develpoing Indigenous competencies in higher education. Journal of University Teaching \& Learning Practice, 13(2), 1-19.

Boutin-Foster, C., Foster, J. C., \& Konopasek, L. (2008). Physician, know thyself: The professional culture of medicine as a framework for teaching cultural competence. Academic Medicine, 83 (1), 106-111.

Braun, V., \& Clarke, V. (2013). Successful qualitative research: A practical guide for beginners. Los Angeles, CA: Sage.

Bringle, R. G., Hatcher, J., \& Jones, S. (Eds.). (2011). International service learning: Conceptual frameworks and research. Sterling, VA: Stylus.

Commonwealth of Australia_-Department of Health. (2014). Appendix B: Developing Aboriginal and Torres Strait Islander cultural capabilities in health graduates: A review of the literature. Canberra, ACT, Australia: Health Workforce Australia.

Culture. (2018). In Cambridge Dictionary. Retrieved from https://dictionary. cambridge.org/dictionary/english/culture

Downing, R., Kowal, E., \& Paradies, Y. (2011). Indigenous cultural training for health workers in Australia. International Journal for Quality in Health Care, 23(3), 247-257.

Durey, A. (2010). Reducing racism in Aboriginal health care in Australia: Where does cultural education fit? Australian and New Zealand Journal of Public Health, 34(Suppl. 1), S87-S92.

Gladman, J., Ryder, C., \& Walters, L. K. (2015). Measuring organisational-level Aboriginal cultural climate to tailor cultural safety strategies. Rural and Remote Health, 15(4), 3050.

Laverty, M., McDermott, D. R., \& Calma, T. (2017). Embedding cultural safety in Australia's main health care standards. Medical Journal of Australia, 207(1), 15-16.

Phillips, G. (2004). CDAMS Indigenous health curriculum framework. Retrieved from https://medicaldeans.org.au/md/2018/07/cdams-indig-health-frameworkreport. pdf

Roberts, J. H., Sanders, T., Mann, K., \& Wass, V. (2010). Institutional marginalisation and student resistance: Barriers to learning about culture, race and ethnicity. Advances in Health Science Education, 15, 559-571. 